\title{
POSITIVE SOLUTIONS OF FUNCTIONAL DIFFERENCE EQUATIONS WITH $p$-LAPLACIAN OPERATOR
}

\author{
CHANG-XIU SONG
}

Received 18 October 2005; Accepted 10 January 2006

The author studies the boundary value problems with $p$-Laplacian functional difference equation $\triangle \phi_{p}(\triangle x(t))+r(t) f\left(x_{t}\right)=0, t \in[0, N], x_{0}=\psi \in C^{+}, x(0)-B_{0}(\triangle x(0))=0$, $\triangle x(N+1)=0$. By using a fixed point theorem in cones, sufficient conditions are established for the existence of twin positive solutions.

Copyright (c) 2006 Chang-Xiu Song. This is an open access article distributed under the Creative Commons Attribution License, which permits unrestricted use, distribution, and reproduction in any medium, provided the original work is properly cited.

\section{Introduction}

For notation, given $a<b$ in $\mathbb{Z}$, we employ intervals to denote discrete sets such as $[a, b]=$ $\{a, a+1, \ldots, b\},[a, b)=\{a, a+1, \ldots, b-1\},[a, \infty)=\{a, a+1, \ldots\}$, and so forth. Let $\tau, N \in$ $\mathbb{Z}$ and let $0 \leq \tau \leq N$. In this paper, we are concerned with the $p$-Laplacian functional difference equation

$$
\begin{gathered}
\triangle \phi_{p}(\triangle x(t))+r(t) f\left(x_{t}\right)=0, \quad t \in[0, N], \\
x_{0}=\psi \in C^{+}, \quad x(0)-B_{0}(\triangle x(0))=0, \quad \triangle x(N+1)=0,
\end{gathered}
$$

where $\phi_{p}(u)$ is the $p$-Laplacian operator, that is, $\phi_{p}(u)=|u|^{p-2} u, p>1,\left(\phi_{p}\right)^{-1}(u)=$ $\phi_{q}(u), 1 / p+1 / q=1$. For all $t \in \mathbb{Z}$, let $x_{t}=x_{t}(k)=x(t+k), k \in[-\tau,-1]$; then $x_{t} \in C$, where $C=C([-\tau,-1], \mathbb{R})$ is a Banach space with the norm $\|\varphi\|_{C}=\max _{k \in[-\tau,-1]}|\varphi|$. Let $C^{+}=\{\varphi \in C: \varphi(k) \geq 0, k \in[-\tau,-1]\}$ and let $d=\max _{k \in[-\tau,-1]} \psi(k), \psi \in C^{+}$. As usual, $\triangle$ denotes the forward difference operator defined by $\triangle x(t)=x(t+1)-x(t)$.

We will assume that

$\left(\mathrm{H}_{1}\right) f(\varphi)$ is a nonnegative continuous functional defined on $\mathrm{C}^{+}$;

$\left(\mathrm{H}_{2}\right) r(t)$ is a nonnegative function defined on $[0, N]$;

$\left(\mathrm{H}_{3}\right) B_{0}: \mathbb{R} \rightarrow \mathbb{R}$ is continuous and satisfies that there are $\beta \geq \alpha \geq 0$ such that $\alpha s \leq$ $B_{0}(s) \leq \beta s$ for $s \in \mathbb{R}^{+}$, where $\mathbb{R}^{+}$denotes the set of nonnegative real numbers. 
Recently, the existence of positive solutions of finite difference equations with different boundary value conditions is investigated in [1-5] and references therein. In this paper, we consider the functional difference equation (1.1) and apply the twin fixed point theorem to obtain at least two positive solutions of the boundary value problem (BVP) (1.1) when growth conditions are imposed on $f$. Finally, we present two corollaries that show that under the assumptions that $f$ is superlinear or sublinear, BVP (1.1) has at least two positive solutions. An example to illustrate our results in this paper is included.

We note that $x(t)$ is a solution of (1.1) if and only if

$$
x(t)= \begin{cases}B_{0}\left(\phi_{q}\left(\sum_{n=0}^{N} r(n) f\left(x_{n}\right)\right)\right)+\sum_{m=0}^{t-1} \phi_{q}\left(\sum_{n=m}^{N} r(n) f\left(x_{n}\right)\right), & t \in[0, N+2], \\ \psi, & t \in[-\tau,-1] .\end{cases}
$$

We assume that $\bar{x}(t)$ is the solution of BVP (1.1) with $f \equiv 0$. Clearly, it can be expressed as

$$
\bar{x}(t)= \begin{cases}0, & t \in[0, N+2], \\ \psi, & t \in[-\tau,-1] .\end{cases}
$$

It is obvious that $\bar{x}_{n} \equiv 0$ for $n \in[\tau, N]$.

Let $x(t)$ be a solution of BVP $(1.1)$ and $y(t)=x(t)-\bar{x}(t)$. Noting that $y(t)=x(t)$ for $t \in[0, N+2]$, then we have from (1.2) that

$$
y(t)= \begin{cases}B_{0}\left(\phi_{q}\left(\sum_{n=0}^{N} r(n) f\left(y_{n}+\bar{x}_{n}\right)\right)\right)+\sum_{m=0}^{t-1} \phi_{q}\left(\sum_{n=m}^{N} r(n) f\left(y_{n}+\bar{x}_{n}\right)\right), & t \in[0, N+2], \\ 0, & t \in[-\tau,-1] .\end{cases}
$$

Let $E=\{y:[-\tau, N+2] \rightarrow \mathbb{R}\}$ with norm $\|y\|=\max _{t \in[-\tau, N+2]}|y(t)|$, then $(E,\|\cdot\|)$ is a Banach space.

Define a cone $P$ by

$$
\begin{aligned}
& P=\{y \in E: y(t)=0 \text { for } t \in[-\tau,-1] ; y(t) \geq 0 \text { for } t \in[0, N+2], \\
&\text { and } \left.\triangle^{2} y(t) \leq 0, \triangle y(t) \geq 0 \text { for } t \in[0, N+2], \triangle y(N+1)=0\right\} .
\end{aligned}
$$

Clearly, $\|y\|=\|y\|_{[0, N+2]}=y(N+2)$ for $y(t) \in P$, where $\|y\|_{[0, N+2]}=\max _{t \in[0, N+2]}|y(t)|$. Define $T: P \rightarrow E$ by

$T y(t)$

$$
= \begin{cases}B_{0}\left(\phi_{q}\left(\sum_{n=0}^{N} r(n) f\left(y_{n}+\bar{x}_{n}\right)\right)\right)+\sum_{m=0}^{t-1} \phi_{q}\left(\sum_{n=m}^{N} r(n) f\left(y_{n}+\bar{x}_{n}\right)\right), & t \in[0, N+2], \\ 0, & t \in[-\tau,-1] .\end{cases}
$$


The following lemma will play an important role in the proof of our results and can be found in [2]. Let

$$
\begin{aligned}
P(\delta, e) & =\{x \in P: \delta(x)<e\}, \\
\partial P(\delta, e) & =\{x \in P: \delta(x)=e\}, \\
\overline{P(\delta, e)} & =\{x \in P: \delta(x) \leq e\} .
\end{aligned}
$$

Lemma 1.1. Let $X$ be a real Banach space, $P$ a cone of $X, \gamma$ and $\alpha$ two nonnegative increasing continuous maps, $\theta$ a nonnegative continuous map, and $\theta(0)=0$. There are two positive numbers $c$ and $M$ such that

$$
\gamma(x) \leq \theta(x) \leq \alpha(x), \quad\|x\| \leq M \gamma(x) \quad \text { for } x \in \overline{P(\gamma, c)} .
$$

In addition, assume that $T: \overline{P(\gamma, c)} \rightarrow P$ is completely continuous. There are positive numbers $0<a<b<c$ such that

$$
\theta(\lambda x) \leq \lambda \theta(x) \quad \forall \lambda \in[0,1], x \in \partial P(\theta, b),
$$

and

(i) $\gamma(T x)>c$ for $x \in \partial P(\gamma, c)$;

(ii) $\theta(T x)<b$ for $x \in \partial P(\theta, b)$;

(iii) $\alpha(T x)>a$ and $P(\alpha, a) \neq \varnothing$ for $x \in \partial P(\alpha, a)$.

Then $T$ has at least two fixed points $x_{1}$ and $x_{2} \in \overline{P(\gamma, c)}$ satisfying

$$
a<\alpha\left(x_{1}\right), \quad \theta\left(x_{1}\right)<b, \quad b<\theta\left(x_{2}\right), \quad \gamma\left(x_{2}\right)<c .
$$

The following lemma is similar to Lemma 1.1; the proof is omitted.

Lemma 1.2. Let $X$ be a real Banach space, $P$ a cone of $X, \gamma$ and $\alpha$ two nonnegative increasing continuous maps, $\theta$ a nonnegative continuous map, and $\theta(0)=0$. There are two positive numbers $c$ and $M$ such that

$$
\gamma(x) \leq \theta(x) \leq \alpha(x), \quad\|x\| \leq M \gamma(x) \quad \text { for } x \in \overline{P(\gamma, c)} .
$$

In addition, assume that $T: \overline{P(\gamma, c)} \rightarrow P$ is completely continuous. There are positive numbers $0<a<b<c$ such that

$$
\theta(\lambda x) \leq \lambda \theta(x) \quad \forall \lambda \in[0,1], x \in \partial P(\theta, b)
$$

and

(i) $\gamma(T x)<c$ for $x \in \partial P(\gamma, c)$;

(ii) $\theta(T x)>b$ for $x \in \partial P(\theta, b)$;

(iii) $\alpha(T x)<a$ and $P(\alpha, a) \neq \varnothing$ for $x \in \partial P(\alpha, a)$.

Then $T$ has at least two fixed points $x_{1}$ and $x_{2} \in \overline{P(\gamma, c)}$ satisfying

$$
a<\alpha\left(x_{1}\right), \quad \theta\left(x_{1}\right)<b, \quad b<\theta\left(x_{2}\right), \quad \gamma\left(x_{2}\right)<c .
$$


4 Positive solutions of difference equations

\section{Main results}

Choose $h=[(N+2) / 2]$, where $[x]$ is the greatest integer not greater than $x$.

Lemma 2.1. Let $T$ be defined by (1.4). If $y \in P$, then

(i) $T(P) \subset P$;

(ii) $T: P \rightarrow P$ is completely continuous;

(iii) finding positive solutions of BVP (1.1) is equivalent to finding fixed points of the operator $T$ on $P$;

(iv) if $y \in P$, then

$$
y(t) \geq \frac{1}{2}\|y\|=\frac{1}{2} y(N+2), \quad t \in[h, N+2] .
$$

The proof is simple and is omitted.

Define the nonnegative, increasing, continuous functionals $\gamma, \theta$, and $\alpha$ on $P$ by

$$
\begin{gathered}
\gamma(y)=y(h), \\
\theta(y)=\max _{t \in[0, h]} y(t)=y(h), \\
\alpha(y)=\max _{t \in[0, h]} y(t)=y(h) .
\end{gathered}
$$

We have

$$
\begin{gathered}
\gamma(y)=\theta(y)=\alpha(y), \quad y \in P, \\
\theta(y)=\gamma(y)=y(h) \geq\left(\frac{1}{2}\right) y(N+2)=\left(\frac{1}{2}\right)\|y\| \text { for each } y \in P .
\end{gathered}
$$

Then

$$
\begin{gathered}
\|y\| \leq 2 \gamma(y), \quad \text { for each } y \in P, \\
\theta(\lambda y)=\lambda \theta(y), \quad \forall \lambda \in[0,1], y \in \partial P(\theta, b) .
\end{gathered}
$$

For the notational convenience, we denote $\sigma$ and $\rho$ by

$$
\begin{aligned}
& \sigma=(\alpha+1) \phi_{q}\left(\sum_{n=h+\tau}^{N} r(n)\right) \\
& \rho=(\beta+h) \phi_{q}\left(\sum_{n=0}^{N} r(n)\right) .
\end{aligned}
$$

Throughout the paper, we assume that $h+\tau \leq N$ and $\sum_{n=h+\tau}^{N} r(n)>0$.

Theorem 2.2. Suppose that there are positive numbers $a<b<c$ such that

$$
0<a<\frac{\sigma}{\rho} b<\frac{\sigma}{2 \rho}(c-d)
$$


Assume that $f(\varphi)$ satisfies the following conditions:

(A) $f(\varphi)>\phi_{p}(c / \sigma)$ for $c \leq\|\varphi\|_{C} \leq 2 c$,

(B) $f(\varphi)<\phi_{p}(b / \rho)$ for $0 \leq\|\varphi\|_{C} \leq 2 b+d$,

(C) $f(\varphi)>\phi_{p}(a / \sigma)$ for $a \leq\|\varphi\|_{C} \leq 2 a$.

Then BVP (1.1) has at least two positive solutions $x_{1}$ and $x_{2}$ such that

$$
a<\max _{t \in[0, h]} x_{1}(t)<b<\max _{t \in[0, h]} x_{2}(t)<c .
$$

Proof. Firstly, we verify that $y \in \partial P(\gamma, c)$ implies that $\gamma(T y)>c$.

Since $\gamma(y)=c=y(h)$, one gets $y(t) \geq c$ for $t \in[h, N+2]$.

Recalling that $\|y\| \leq 2 \gamma(y)=2 c$, we know that $c \leq\left\|y_{n}\right\|_{C} \leq 2 c$ for $n \in[h+\tau, N]$. Then, we get

$$
\begin{aligned}
\gamma(T y) & =B_{0}\left(\phi_{q}\left(\sum_{n=0}^{N} r(n) f\left(y_{n}+\bar{x}_{n}\right)\right)\right)+\sum_{m=0}^{h-1} \phi_{q}\left(\sum_{n=m}^{N} r(n) f\left(y_{n}+\bar{x}_{n}\right)\right) \\
& \geq \alpha \phi_{q}\left(\sum_{n=0}^{N} r(n) f\left(y_{n}+\bar{x}_{n}\right)\right)+\sum_{m=0}^{h-1} \phi_{q}\left(\sum_{n=m}^{N} r(n) f\left(y_{n}+\bar{x}_{n}\right)\right) \\
& \geq \alpha \phi_{q}\left(\sum_{n=h+\tau}^{N} r(n) f\left(y_{n}\right)\right)+\phi_{q}\left(\sum_{n=h+\tau}^{N} r(n) f\left(y_{n}\right)\right) \\
& =(\alpha+1) \phi_{q}\left(\sum_{n=h+\tau}^{N} r(n) f\left(y_{n}\right)\right)>(\alpha+1) \phi_{q}\left(\sum_{n=h+\tau}^{N} r(n) \phi_{p}\left(\frac{c}{\sigma}\right)\right) \\
& =\frac{c}{\sigma}(\alpha+1) \phi_{q}\left(\sum_{n=h+\tau}^{N} r(n)\right)=c .
\end{aligned}
$$

Secondly, we prove that $y \in \partial P(\theta, b)$ implies that $\theta(T y)<b$.

Since $\theta(y)=b$ implies that $y(h)=b$, it follows that $0 \leq y(t) \leq b$ for $t \in[0, h]$ and

$$
b \leq y(t) \leq\|y\| \leq 2 \theta(y)=2 b, \quad \text { for } t \in[h+1, N], y \in P .
$$

So

$$
\left\|y_{n}+\bar{x}_{n}\right\|_{C} \leq\left\|y_{n}\right\|_{C}+\left\|\bar{x}_{n}\right\|_{C} \leq 2 b+d
$$

Then, we have

$$
\begin{aligned}
\theta(T y) & =B_{0}\left(\phi_{q}\left(\sum_{n=0}^{N} r(n) f\left(y_{n}+\bar{x}_{n}\right)\right)\right)+\sum_{m=0}^{h-1} \phi_{q}\left(\sum_{n=m}^{N} r(n) f\left(y_{n}+\bar{x}_{n}\right)\right) \\
& <\beta \phi_{q}\left(\sum_{n=0}^{N} r(n) f\left(y_{n}+\bar{x}_{n}\right)\right)+\sum_{m=0}^{h-1} \phi_{q}\left(\sum_{n=0}^{N} r(n) f\left(y_{n}+\bar{x}_{n}\right)\right) \\
& =\frac{b}{\rho}(\beta+h) \phi_{q}\left(\sum_{n=0}^{N} r(n)\right)=b .
\end{aligned}
$$


Finally, we show that

$$
P(\alpha, a) \neq \varnothing, \quad \alpha(T y)>a \quad \forall y \in \partial P(\alpha, a)
$$

It is obvious that $P(\alpha, a) \neq \varnothing$. On the other hand, $\alpha(y)=y(h)=a$ implies that

$$
\begin{gathered}
a \leq\|y\| \leq 2 a \quad \text { for } t \in[h, N], \\
a \leq\left\|y_{n}\right\|_{C} \leq 2 a \quad \text { for } n \in[h+\tau, N] .
\end{gathered}
$$

Thus,

$$
\begin{aligned}
\alpha(T y) & =B_{0}\left(\phi_{q}\left(\sum_{n=0}^{N} r(n) f\left(y_{n}+\bar{x}_{n}\right)\right)\right)+\sum_{m=0}^{h-1} \phi_{q}\left(\sum_{n=m}^{N} r(n) f\left(y_{n}+\bar{x}_{n}\right)\right) \\
& \geq \alpha \phi_{q}\left(\sum_{n=0}^{N} r(n) f\left(y_{n}+\bar{x}_{n}\right)\right)+\sum_{m=0}^{h-1} \phi_{q}\left(\sum_{n=m}^{N} r(n) f\left(y_{n}+\bar{x}_{n}\right)\right) \\
& \geq \alpha \phi_{q}\left(\sum_{n=h+\tau}^{N} r(n) f\left(y_{n}\right)\right)+\phi_{q}\left(\sum_{n=h+\tau}^{N} r(n) f\left(y_{n}\right)\right) \\
& =(\alpha+1) \phi_{q}\left(\sum_{n=h+\tau}^{N} r(n) f\left(y_{n}\right)\right)>(\alpha+1) \phi_{q}\left(\sum_{n=h+\tau}^{N} r(n) \phi_{p}\left(\frac{a}{\sigma}\right)\right) \\
& =\frac{a}{\sigma}(\alpha+1) \phi_{q}\left(\sum_{n=h+\tau}^{N} r(n)\right)=a .
\end{aligned}
$$

Hence by Lemma 1.1, $T$ has at least two different fixed points $y_{1}$ and $y_{2}$. Let $x_{i}=y_{i}+\bar{x}$ $(i=1,2)$, which are twin positive solutions of BVP (1.1) such that (2.7) holds. The proof is complete.

Theorem 2.3. Suppose that there are positive numbers $0<a<b<c$ such that

$$
0<2 a+d<b<\frac{\sigma}{\rho} c
$$

Assume that $f(\varphi)$ satisfies the following conditions:

$\left(\mathrm{A}^{\prime}\right) f(\varphi)<\phi_{p}(c / \rho)$ for $0 \leq\|\varphi\|_{C} \leq 2 c+d$,

(B') $f(\varphi)>\phi_{p}(b / \sigma)$ for $b \leq\|\varphi\|_{C} \leq 2 b$,

$\left(\mathrm{C}^{\prime}\right) f(\varphi)<\phi_{p}(a / \rho)$ for $0 \leq\|\varphi\|_{C} \leq 2 a+d$.

Then BVP (1.1) has at least two positive solutions $x_{1}$ and $x_{2}$ such that

$$
a<\max _{t \in[0, h]} x_{1}(t)<b<\max _{t \in[0, h]} x_{2}(t)<c
$$

The proof is omitted since it is similar to that of Theorem 2.2.

Now, we give theorems which may be considered as the corollaries of Theorems 2.2 and 2.3. 
Let

$$
f_{0}=\lim _{\|\varphi\|_{C} \rightarrow 0} \frac{f(\varphi)}{\|\varphi\|_{C}^{p-1}} ; \quad f_{\infty}=\lim _{\|\varphi\|_{C} \rightarrow \infty} \frac{f(\varphi)}{\|\varphi\|_{C}^{p-1}}
$$

and choose $k_{1}, k_{2}, k_{3}$ such that

$$
k_{i} \sigma>1, \quad i=1,2,0<k_{3} \rho<1 \text {. }
$$

THEOREM 2.4. Let the following conditions be satisfied:

(D) $f_{0}>k_{1}{ }^{p-1}, f_{\infty}>k_{2}{ }^{p-1}$;

(E) there exists a $p_{1}>0$ such that for $0 \leq\|\varphi\|_{C} \leq 2 p_{1}+d$, one has $f(\varphi)<\left(p_{1} / \rho\right)^{p-1}$.

Then BVP (1.1) has at least two positive solutions.

Proof. Firstly, choose $b=p_{1}$, then

$$
f(\varphi)<\left(\frac{2 p_{1}}{\rho}\right)^{p-1}=\phi_{p}\left(\frac{b}{\rho}\right) \text { for } 0 \leq\|\varphi\|_{C} \leq 2 b+d .
$$

Secondly, since $f_{0}>k_{1}^{p-1}$, there is $R_{1}>0$ sufficiently small such that

$$
f(\varphi)>\left(k_{1}\|\varphi\|_{C}\right)^{p-1} \quad \text { for } 0 \leq\|\varphi\|_{C} \leq R_{1} .
$$

Without loss of generality, suppose that

$$
R_{1} \leq \frac{2 \sigma}{\rho} b
$$

Choose $a>0$ so that $a<(1 / 2) R_{1}$. For $a \leq\|\varphi\|_{C} \leq 2 a$, we have $\|\varphi\|_{C} \leq R_{1}$ and $a<(\sigma / \rho) b$. Thus,

$$
f(\varphi)>\left(k_{1}\|\varphi\|_{C}\right)^{p-1} \geq\left(k_{1} a\right)^{p-1}>\phi_{p}\left(\frac{a}{\sigma}\right) \quad \text { for } a \leq\|\varphi\|_{C} \leq 2 a .
$$

Thirdly, since $f_{\infty}>k_{2}{ }^{p-1}$, there is $R_{2}>0$ sufficiently large such that

$$
f(\varphi)>\left(k_{2}\|\varphi\|_{C}\right)^{p-1} \text { for }\|\varphi\|_{C} \geq R_{2}
$$

Without loss of generality, suppose that $R_{2}>2 b$. Choose $c \geq R_{2}+d$. Then,

$$
f(\varphi)>\left(k_{2}\|\varphi\|_{C}\right)^{p-1} \geq\left(k_{2} c\right)^{p-1}>\phi_{p}\left(\frac{c}{\sigma}\right) \text { for } c \leq\|\varphi\|_{C} \leq 2 c .
$$

We then have $0<a<(\sigma / \rho) b<(\sigma / 2 \rho)(c-d)$, and now the conditions in Theorem 2.2 are all satisfied. By Theorem 2.2, BVP (1.1) has at least two positive solutions. The proof is complete. 


\section{Positive solutions of difference equations}

THEOREM 2.5. Let the following conditions be satisfied:

(F) $f_{0}<k_{3}^{p-1}$;

(G) there exists a $p_{2}>0$ such that for $0 \leq\|\varphi\|_{C} \leq 2 p_{2}$, one has $f(\varphi)>\left(p_{2} / \sigma\right)^{p-1}$.

Then BVP (1.1) has at least two positive solutions.

The following corollaries are obvious.

Corollary 2.6. Let the following conditions be satisfied:

$\left(\mathrm{D}^{\prime}\right) f_{0}=\infty, f_{\infty}=\infty$;

(E) there exists a $p_{1}>0$ such that for $0 \leq\|\varphi\|_{C} \leq 2 p_{1}+d$, one has $f(\varphi)<\left(p_{1} / \rho\right)^{p-1}$.

Then BVP (1.1) has at least two positive solutions.

Corollary 2.7. Let the following conditions be satisfied:

$\left(\mathrm{F}^{\prime}\right) f_{0}=0$

$(\mathrm{G})$ there exists a $p_{2}>0$ such that for $0 \leq\|\varphi\|_{C} \leq 2 p_{2}$, one has $f(\varphi)>\left(p_{2} / \sigma\right)^{p-1}$.

Then BVP (1.1) has at least two positive solutions.

\section{Example}

Example 3.1. Consider BVP

$$
\begin{gathered}
\triangle \phi_{p}(\triangle x(t))+r\left[x^{1 / 9}(t-1)+x^{1 / 3}(t-1)\right]=0, \quad t \in[0,4], \\
x(t)=\psi(t), \quad t=-1, x(0)=0, x(5)=x(6)=1,
\end{gathered}
$$

where $\tau=1, k=-1, N=4, h=3, \alpha=\beta=0, r>0$ is a constant satisfying $\sum_{n=h+\tau}^{N} r>0$, $\psi(t) \geq 0, d=\|\psi\|_{C}=\max _{k=-1}|\psi(k)|>0, p=7 / 6, q=7$, and $f(\varphi)=\varphi^{1 / 9}(-1)+\varphi^{1 / 3}(-1)$.

Suppose that $\varphi \in C^{+}$, then $\|\varphi\|_{C}=\varphi(-1)$.

As $\|\varphi\|_{C} \rightarrow 0$ or $\|\varphi\|_{C} \rightarrow+\infty$, we get

$$
\begin{aligned}
\frac{f(\varphi)}{\|\varphi\|_{C}^{p-1}} & =\frac{\varphi^{1 / 9}(-1)+\varphi^{1 / 3}(-1)}{\|\varphi\|_{C}^{p-1}} \\
& =\|\varphi\|_{C}^{(10-9 p) / 9}+\|\varphi\|_{C}^{(4-3 p) / 3} \longrightarrow+\infty .
\end{aligned}
$$

We deduce that

$$
\rho=(\beta+h) \phi_{q}\left(\sum_{n=0}^{N} r(n)\right)=3\left[\sum_{n=0}^{4} r\right]^{6}=46875 r,
$$

thus, for all $m>0$ and $0 \leq\|\varphi\|_{C} \leq m+d$, one has

$$
0 \leq f(\varphi) \leq(m+d)^{1 / 9}+(m+d)^{1 / 3}=(m+d)^{1 / 9}\left(m^{1-p}+\frac{(m+d)^{2 / 9}}{m^{p-1}}\right) m^{p-1} .
$$

Define $H(m)=(m+d)^{1 / 9}\left(m^{1-p}+(m+d)^{2 / 9} / m^{p-1}\right)$.

Suppose that $r$ and $d$ satisfy

$$
(2 d)^{1 / 9}\left(d^{-1 / 6}+2^{2 / 9} d^{1 / 18}\right)<\left(\frac{1}{2 \rho}\right)^{p-1} ;
$$


then $H(d)=(2 d)^{1 / 9}\left(d^{-1 / 6}+2^{2 / 9} d^{1 / 18}\right)<(1 / 2 \rho)^{p-1}$ holds. So, we can find a $p_{1}=d / 2$ such that $f(\varphi) \leq H\left(2 p_{1}\right)\left(2 p_{1}\right)^{p-1}<\left(p_{1} / \rho\right)^{p-1}$ for $0 \leq\|\varphi\|_{C} \leq 2 p_{1}+d$. By Corollary 2.6, we know that BVP (3.1) has at least two positive solutions.

\section{Acknowledgment}

This research was supported by Natural Science Foundation of Guangdong Province (011471), China.

\section{References}

[1] R. P. Agarwal and J. Henderson, Positive solutions and nonlinear eigenvalue problems for thirdorder difference equations, Computers \& Mathematics with Applications 36 (1998), no. 10-12, 347-355.

[2] R. I. Avery, C. J. Chyan, and J. Henderson, Twin solutions of boundary value problems for ordinary differential equations and finite difference equations, Computers \& Mathematics with Applications 42 (2001), no. 3-5, 695-704.

[3] A. Cabada, Extremal solutions for the difference $\phi$-Laplacian problem with nonlinear functional boundary conditions, Computers \& Mathematics with Applications 42 (2001), no. 3-5, 593601.

[4] J. Henderson, Positive solutions for nonlinear difference equations, Nonlinear Studies 4 (1997), no. 1,29-36.

[5] Y. Liu and W. Ge, Twin positive solutions of boundary value problems for finite difference equations with p-Laplacian operator, Journal of Mathematical Analysis and Applications 278 (2003), no. 2, 551-561.

Chang-Xiu Song: School of Mathematical Sciences, South China Normal University, Guangzhou 510631, China

Current address: School of Applied Mathematics, Guangdong University of Technology,

Guangzhou 510006, China

E-mail address: scx168@sohu.com 